\title{
Association study of miR-22 and miR-335 expression levels and G2 assay related inherent radiosensitivity in peripheral blood of ductal carcinoma breast cancer patients
}

\author{
Narjes BAKHTARI ${ }^{1}$, Hossein MOZDARANI ${ }^{1, *}$, Mahdieh SALIMI ${ }^{2}$, Ramesh OMRANIPOUR ${ }^{3}$ \\ ${ }^{1}$ Department of Medical Genetics, Faculty of Medical Sciences, Tarbiat Modares University, Tehran, Iran; ${ }^{2}$ Department of Medical Genetics, \\ Medical Biotechnology Institute, National Institute of Genetic Engineering and Biotechnology, Tehran, Iran; ${ }^{3}$ Cancer Institute, Tehran University \\ of Medical Sciences, Tehran, Iran
}

*Correspondence: mozdarah@modares.ac.ir

Received February 25, 2020 / Accepted June 2, 2020

\begin{abstract}
Identifying patient's cellular radiosensitivity before radiotherapy (RT) in breast cancer (BC) patients allows proper alternations in routinely used treatment programs and reduces the adverse side effects in exposed patients. This study was conducted on blood samples taken from 60 women diagnosed with Invasive Ductal Carcinoma (IDC) BC (mean age: $47 \pm 9.93$ ) and 30 healthy women (mean age: $44.43 \pm 6.7$ ). The standard G2 assay was performed to predict cellular radiosensitivity. To investigate miR-22 and miR-335 expression levels in peripheral blood mononuclear cells (PBMCs), qPCR was performed. The sensitivity and specificity of the mentioned miRNAs were assessed by plotting the Receiver Operating Characteristic (ROC) curve. Binary logistic regression was performed to identify the miRNA involvement in $\mathrm{BC}$ and cellular radiosensitivity (CR) of BC patients. The frequency of spontaneous and radiation-induced chromatid breaks (CBs) was significantly different between control and patient groups $(\mathrm{p}<0.05)$. A cut-off value was determined to differentiate the patients with and without cellular radiosensitivity. miR-22 and miR-335 were significantly downregulated in BC patients. miRNAs expression levels were directly associated with CR. ROC curve assessment identified that both miRNAs had acceptable specificity and sensitivity in the prediction of BC and CR of BC patients. Binary logistic regression showed that both miRNAs could also predict BC successfully. Although only miR-22 was shown potent to predict CR of BC patients, both miR-22 and miR-335 might act as tumor suppressor miRNAs in BC. miR-22 and miR-335 may be promising potential biomarkers in $\mathrm{BC}$ prediction along with other important biomarkers. Moreover, mirR-22 might be a potential biomarker for the prediction of $\mathrm{CR}$ in $\mathrm{BC}$ patients.
\end{abstract}

Key words: breast cancer, G2 assay, cellular radiosensitivity, miR-22, miR-335

Breast cancer (BC), is the most common type of cancer among women and the first cause of cancer-related mortality in women worldwide [1]. Radiotherapy (RT) is a common method used for the treatment of $\sim 50 \%$ of all cancer patients in some stages of their disease [2]; however, some patients are over/undertreated after RT [3]. Radiosensitivity means the relative sensitivity of normal cells, tissues, or organs to the effects of ionizing radiation [4]. Cellular radiosensitivity depends on several factors including the type of radiation, the DNA repair capacity, etc. [5]. Identifying radiosensitive patients before performing RT allows a proper alternation in routinely used treatment regimens to reduce the adverse side effects in exposed patients [4]. Radiation response analysis in different subtypes of breast cancer has revealed that luminal subtypes $(A, B)$ are more sensitive to irradiation although triple negative breast cancers (TNBC) and Her2+ subtypes are almost radio-resistant [6]. Provided that distinct molecular subtypes of $\mathrm{BC}$ show a different response to irradiation, it might affect the clinical outcome of treatment [7].

Studies have also revealed that peripheral blood lymphocytes from patients with different types of cancer show higher chromosomal abnormalities (CA) than healthy individuals after irradiation [8]. Enhanced CA after irradiation has been detected in $\sim 40 \%$ of BC patients while only in $\sim 10 \%$ of healthy individuals $[9,10]$. G2 assay, a widely used method for the study of radiosensitivity, is in vitro irradiation of peripheral blood lymphocytes in the G2 phase of the cell cycle to create DNA damage, which is often repaired during G2 to M-phase transition, residual lesions can be observed and measured at metaphase as CA [11]. The high 
frequencies of chromosomal aberrations expressed following G2 exposure significantly differentiate between radiosensitive and non-radiosensitive cells or individuals [12-16]. Several reports have shown higher frequency of chromosomal aberrations in lymphocytes of breast cancer following G2 exposure to ionizing radiation [17-19]. Ionizing radiation (IR) used in RT, induces several types of DNA lesions such as double-strand breaks (DSBs) [20], DNA doublestrand breaks (DSBs) are a hazardous form of damage that can potentially cause cell death or genomic rearrangements. DSBs are repaired with two-component kinetics. In both phases, a fast process uses canonical nonhomologous end-joining (c-NHEJ) to repair the majority of DSBs [21]. Chromatid aberrations in G2 may be induced following a signaling pathway induced by initial induction of DNA damage $[22,23]$. The DNA damage response (DDR) acts as a critical role in DSBs repair [24]. DDR maintains genomic stability by protecting cells against apoptosis or malignancy [25]. microRNAs (miRNAs) are a subtype of small non-coding RNAs with 20-25 nucleotides length which their role in breast tumorigenesis and modulating radiation response is confirmed [26].

miRNAs can affect tumor radiosensitivity through the regulation of DDR [15]. miR-22 modulates DDR by downregulation of MDC1 expression, MDC1 plays a critical role in genome stability [27]. MDC1 downregulation has been detected in human breast and lung tumor cells [28]. It acts not only as a tumor-suppressor but also as an oncogenic miRNA to stop or aggravate cancer formation [29]. Although, its role in regulating the radiation response of $\mathrm{BC}$ cells is poorly understood its upregulation was shown to increase the radiosensitivity of $\mathrm{BC}$ cells through DNA damage repair [30]. It has been shown that miR-22 acts as a tumorsuppressive miRNA in BC cells [31]. A study showed that the radiotherapy initiates reduction of miR-22 expression [32]. miR-335 regulates the DDR by downregulation of CtIP expression [33]. CtIP regulates DDR through homologous recombination (HR) at the site of DSBs [34]. These findings suggest that miR-335 can sensitize cells to IR [33]. miR-335 also serves as a tumor suppressor miRNA since it has been found to have reduced expression in BC [35]. miR-335 can increase tumor sensitivity to radiation partly via targeting main proteins involved in DDR such as CtIP in breast cancer cell lines but its functions need to be more explored [33].

We aimed to investigate the possible involvement of miR-22 and miR-335 in the radiosensitivity of lymphocytes of IDC BC patients. To do this, a standard G2 assay was used to differentiate patients as radiosensitive and non-radiosensitive groups based on their cellular radiosensitivity. Then the expression levels of miR-22 and miR-335 for the first time in PBMCs was assessed in both patients' group with and without cellular radiosensitivity and compared to the healthy control group. The ultimate aim of this study was to evaluate the potential use of miRNAs used in this study to predict cellular radiosensitivity or early detection of BC.

\section{Patients and methods}

Study population. Stage I/II invasive ductal carcinoma BC patients (60 females; mean age $47 \pm 9.92$ years) were randomly selected at the Cancer Institute of Imam Khomeini Hospital (Tehran, Iran). Patients were selected among new-case individuals who had not received chemotherapy and/or primary radiotherapy treatment or previous anticancer drug before blood sampling. They also had no previous history of alcohol or drug consumption; no history of cancer patients in their first-degree relatives and also signs of congenital chromosomal breakage syndromes associated with radiosensitivity (such as ataxia-telangiectasia, xeroderma pigmentosum, etc.). Age and gender-matched healthy donors (30 females; mean age $44 \pm 6.7$ years) without prior history of breast cancer or other major illnesses in them or their first-degree relatives at the same time were also included as a healthy group. To obtain information on all donors' lifestyles, factors such as dietary habits, medical history, and exposure to chemical and physical agents, smoking, and alcohol/drug consumption, etc. were checked according to written questionnaires. Individuals with these confounding factors for at least one month prior to blood sampling were excluded from the study. Informed consent was obtained from all patients and healthy participants according to the Institutional Ethical Committee regulations. This study was approved by the Ethical Committee of the Tarbiat Modares University (Registration no 52D/1958 dated May 17, 2017).

Cell culture and $\mathrm{G} 2$ chromosomal radiosensitivity assay. Peripheral blood $(2 \mathrm{ml}$ ) from all participants was collected in heparinized tubes. Each blood sample was divided into two parts as non-exposed and exposed to gamma irradiation.

Briefly, $0.5 \mathrm{ml}$ of heparinized blood was added to $4.5 \mathrm{ml}$ of complete RPMI-1640 (Bioidea, EU) supplemented with $10 \%$ fetal bovine serum (Bioidea, EU), penicillin (100 IU/ $\mathrm{ml})$, streptomycin $(100 \mu \mathrm{g} / \mathrm{ml})$ (Bioidea, EU), and $100 \mu \mathrm{l}$ phytohemagglutinin (Gibco, BRL, USA). Each culture vessel was prepared in duplicate, one for checking of spontaneous chromatid breaks yield (SY) as control, and the other for gamma irradiation-induced chromatid breaks yield (IY). Cultures were incubated at $37^{\circ} \mathrm{C}$ for $72 \mathrm{~h}$. Culture vessels were irradiated with a dose of $1 \mathrm{~Gy}$ of gamma-ray generated from a ${ }^{60} \mathrm{Co}$ source (Theratron-II 780C, Kanata, Canada) with a dose rate of $0.8-1 \mathrm{~Gy}$ at room temperature (4-5 h before harvesting). Colcemid (Gibco, BRL) at a concentration of $4 \mu \mathrm{g} / \mathrm{ml}$ was added, $1.5 \mathrm{~h}$ before harvesting to arrest cells at metaphase. Harvesting was performed according to standard procedure, slides were made, and air-dried, then stained in $4 \%$ Giemsa. To analyze metaphase, 100 well spread metaphases were scored in slides before irradiation and 100 metaphases for after irradiation under a light microscope (Leica, Japan) with magnification $\times 1000$ for each sample for the presence of chromatid breaks. The difference between chromatid breaks yield before and after irradiation 

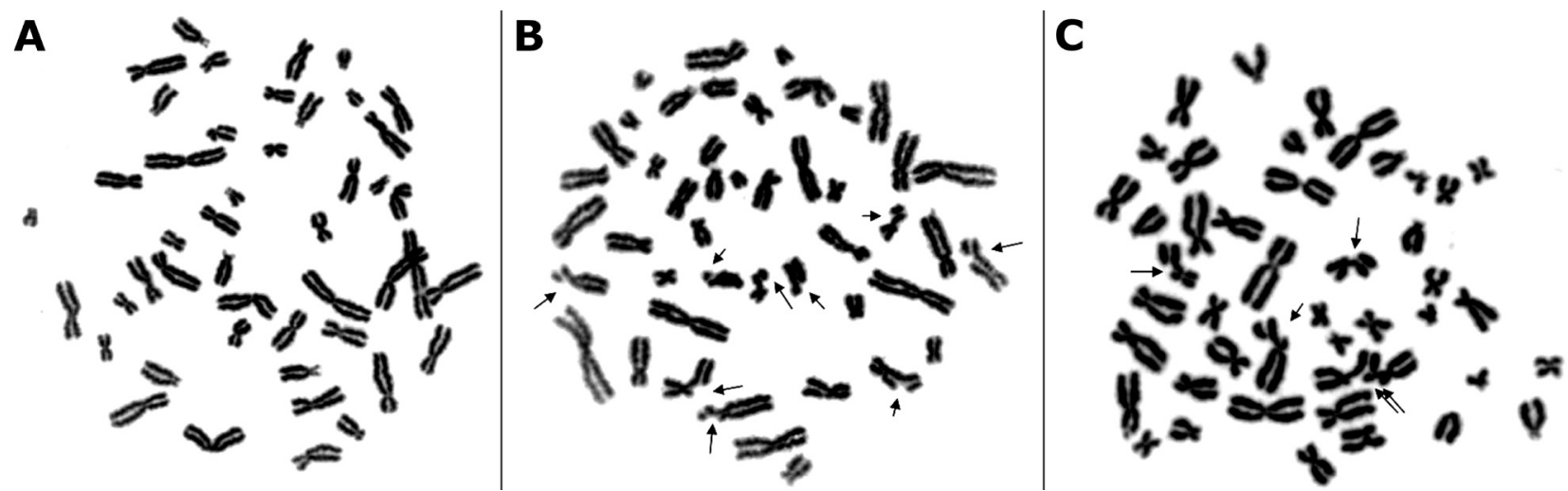

Figure 1. Examples of abnormalities seen in metaphases A) Normal metaphase, B) Metaphase with chromatid breaks and deletions shown with arrows. C) Metaphase with chromatid breaks (shown by arrows) and an exchange (shown by double arrows). Magnification $\times 1000$

was analyzed. Cut-off values were calculated for radiationinduced chromatid aberrations in lymphocytes of healthy individuals as mean aberrations $+1 \mathrm{SD}$ according to Scott et al. $[9,15]$ to differentiate between patients with and without cellular radiosensitivity. Molecular studies were performed according to the cellular radiosensitivity of $\mathrm{BC}$ patients. Figure 1 shows a sample photomicrograph of metaphase cells with and without chromatid aberrations following the irradiation of G2 lymphocytes.

Isolation of peripheral blood mononuclear cells (PBMCs). Two $\mathrm{ml}$ peripheral blood was also collected from each sample in K2-EDTA tubes for molecular experiments. The blood diluted and mixed with an equal volume of phosphate buffer solution (PBS). The solution was gently added to $3 \mathrm{ml}$ Ficoll-Hypaque (Lymphodex Inno-Train) and centrifuged at $400 \mathrm{~g}$ for $40 \mathrm{~min}$. Then the PBMC was carefully transferred into a new tube, and was further diluted in PBS and centrifuged at $500 \mathrm{~g}$ for $15 \mathrm{~min}$ ( 2 times). miRNA isolation procedure was performed on the pellet immediately.

miRNA isolation and qRT-PCR. miRNA was extracted using a kit (FavorPrep ${ }^{\mathrm{TM}}$ miRNA Isolation Kit, Taiwan). To synthesize the cDNA; Reverse transcriptase kit (YTA, Iran) was used and the protocol was followed according to the manufacturer's instruction. Stem loop primers were used to perform reverse transcription. Stem-loop primer sequences used in this study were as follows: hsa-miR-22 (5'-GTCGTATCCAGTGCAGGGTCCGAGGTATTCGCACTGGATACGACACAGTT-3'), hsa-miR-335 (5'-GTCGTATCCAGTGCAGGGTCCGAGGTATTCGCACTGGATACGACACATTT-3'). Hsa-miR-16 was also selected as endogenous control with the following stem-loop primer sequence: (5'-GTCGTATCCAGTGCAGGGTCCGAGGTATTCGCACTGGATACGACCGCCAA-3').

Following reagents: miRNAs, stem-loop RT primer (1 $\mathrm{pM})$, RNase-free water was mixed gently on ice and incubated for $5 \mathrm{~min}$ at $70^{\circ} \mathrm{C}$. Afterward, a mixture containing: $\times 5$ first-

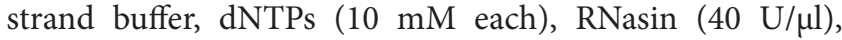
M-MLV was prepared and after adding the mixture into the tubes; incubation was carried out at $16^{\circ} \mathrm{C}$ for $30 \mathrm{~min}$ and $60^{\circ} \mathrm{C}$ for $42 \mathrm{~min}$ using PCR system (Eppendorf). Reaction termination was performed using $5 \mathrm{~min}$ incubation at $70^{\circ} \mathrm{C}$.

Real-time PCR was carried out using SYBR Green qPCR Mix reagent (BIOFACT, Taiwan) on a Real-time PCR System (Applied Biosystems, Step one Plus, USA). The sequences of the specific forward primers were as follows: hsa-miR-22 F (5'-CAGGAGCGAAGCTGCCAGTTGAA-3'), hsa-miR-335 F (5'-GCGGCGGTCAAGAGCAATAACGA-3'), hsa-miR-16 F (5'-GGGCGTTAGCAGCACGTAAA-3'). The universal reverse primer used was (5'-CCAGTGCAGGGTCCGAGGTA-3').

Twenty $\mu$ PCR reaction mixtures included $2 \times$ SYBR Green qPCR Mix reagent (BIOFACT, Taiwan), forward and reverse primer (10 $\mathrm{pM}$ each), cDNA products and nucleasefree water was made. The PCR reactions were incubated at $95^{\circ} \mathrm{C}$ for $10 \mathrm{~min}$, followed by 40 cycles of $95^{\circ} \mathrm{C}$ for $15 \mathrm{sec}$ and $60^{\circ} \mathrm{C}$ for $30 \mathrm{sec}$ and finally $72^{\circ} \mathrm{C}$ for $10 \mathrm{sec}$. All reactions were performed in duplicates. Finally, the $2^{-\Delta \Delta \mathrm{Ct}}$ method was used to analyze the relative expression level of each miRNA.

Statistical analysis. In the G2 assay, for each sample, the SY of chromatid breaks was subtracted from the IY to give the radiation-induced yield (RIY). Results were analyzed using SPSS software (version 25, Chicago, USA). For comparing the frequency of chromatid aberrations between groups before and after irradiation un-paired t-test was used.

The normality of data distribution was investigated using the Shapiro-Wilk test. Outliers were excluded from analysis and the sampling was repeated to obtain $60 \mathrm{BC}$ patients and 30 healthy individuals. The association of miRNAs expression levels with breast cancer was examined by an unpaired t-test (comparison of two groups). Cellular radiosensitivity was analyzed using Tukey Post Hoc One way ANOVA test (comparison of more than 2 groups). A non-parametric Kruskal-Wallis ( $\mathrm{k}$ samples) test was also conducted to determine the significant differences between $\mathrm{BC}$ molecular subtypes. A p-value $<0.05$ was considered as a significant value. 
ROC curves were generated to assess the accuracy of using miRNAs in the detection of breast cancer and cellular radiosensitivity of BC patients. ROC curves and the Area Under the Curve (AUC) with a 95\% confidence interval (95\% $\mathrm{CI})$ were calculated to assess the predictive value of these molecules. Finally, binary logistic regression was employed to evaluate the ability of miRNAs in the prediction of $\mathrm{BC}$ and cellular radiation response.

\section{Results}

Demographic information of patients and their relevant pathological characterization such as the status of ER, PR, Her2, and Ki67 as well as lymph node status and clinical stages are presented in Table 1. The pathological information of some patients was not available.

The G2 assay analysis. The G2 assay was carried out on lymphocytes collected from blood samples of $60 \mathrm{BC}$ women (patients' group) and 30 healthy gender-matched individuals with and without receiving 1 Gy gamma irradiation. The SY was $1 \pm 0.78$ and $3.35 \pm 1.90$ in metaphase related to the lymphocytes of healthy individuals and patients, respectively

Table 1. Demographic information of included patients.

\begin{tabular}{lcc}
\hline Category & Number & Percentage (\%) \\
\hline Age (years): & 18 & 30 \\
$\quad 40 \geq$ & 27 & 45 \\
$54-41$ & 15 & 25 \\
$55<$ & & \\
T Stage ${ }^{\mathrm{a}}$ : & 24 & 40 \\
T1 $\leq 2$ cm & & \\
T2 & 36 & 60 \\
T3 $>2$ cm & & \\
Lymph Node Status ${ }^{\mathrm{b}}:$ & 21 & 35 \\
Positive & 39 & 65 \\
Negative & & \\
Clinical TNM Staging & 17 & 28.33 \\
I & 43 & 71.67 \\
II & & \\
ER/PR/HER2 status: & 16 & 29.63 \\
ER+/PR+/HER2- & 10 & 18.52 \\
ER+/PR-/HER2- & 9 & 16.66 \\
ER-/PR+/HER2- & 3 & 5.56 \\
ER-/PR-/HER2+ & 7 & 12.96 \\
ER+/PR+/HER2+ & 9 & 16.67 \\
ER-/PR-/HER2- & & \\
Mean level of ki-67 & 14 & 40 \\
(Luminal A) $\leq 14$ & 21 & \\
\hline
\end{tabular}

${ }^{\mathrm{a}} \mathrm{T} 0=$ no evidence of primary tumor, $\mathrm{T} 1=$ tumor $\leq 2 \mathrm{~cm}, \mathrm{~T} 2=$ tumor $>2 \mathrm{~cm}$ not exceed from $5 \mathrm{~cm}, \mathrm{~T} 3=$ tumor $>5 \mathrm{~cm}, \mathrm{~T} 4=$ tumors with metastasis to surrounding tissues that were not included in our study; ${ }^{\text {b }}$ ositive $=$ metastasis to axillary lymph nodes and or in internal mammary nodes, negative $=$ no regional lymph node metastasis histologically, no additional examination for isolated tumor cells. 'ER+/PR+/HER2-, ER+/PR-/HER2-, and ER-/PR+/HER2 - are totally considered as BC luminal subtypes. The mean level of Ki-67 is usually determined to differentiate luminal A from luminal $\mathrm{B}$; Abbreviations: $\mathrm{n}=$ number of patients; TNM stage $=$ tumor size; lymph nodes involvement; metastasis; ER = estrogen receptor; HER2 = human epidermal growth factor receptor; $\mathrm{PR}=$ progesterone receptor (unpaired t-test; $\mathrm{p}=0.009$ ). Irradiated yield IY in patients was also significantly different from controls. The average RIY was $1.3 \pm 1.32$ per metaphase in the control group and $7.6 \pm 4.8$ in patients which were significantly higher in patients (unpaired t-test, $\mathrm{p}=0.001$ ). No significant difference was observed in the mean age of these two groups (unpaired t-test, $\mathrm{p}=0.0699$ ).

A cut-off value was obtained to classify the individuals as with cellular radiosensitivity and without cellular radiosensitivity groups, using the mean number of induced chromatid aberrations +1 SD observed for healthy donor's population, according to the method used in the study done by Scott et al. [24]. Results are shown in Figures 2A and 2B.

Based on the determined cut-off value, lymphocyte cultures from patients were classified into 2 groups: with cellular radiosensitivity and without cellular radiosensitivity. From 60 lymphocyte cultures initiated from BC patients and irradiated at G2, thirty-five lymphocyte cultures (58.34\%) showed cellular radiosensitivity, and 25 cultures $(41.66 \%)$ were found without cellular radiosensitivity. For molecular
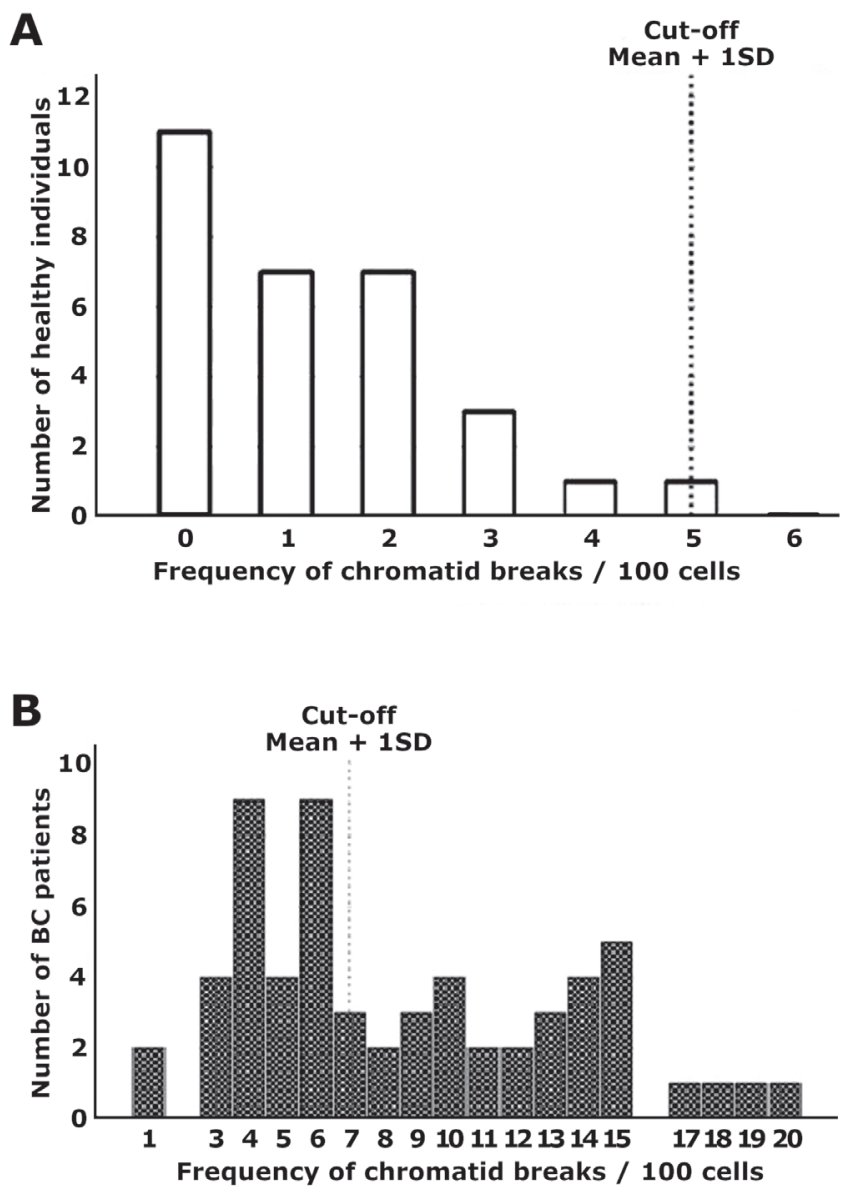

Figure 2. Cut-off values to classify the cells as radiosensitive and nonradiosensitive A) Distribution of radiation-induced chromatid breaks frequency in cells of healthy individuals $(n=30)$ and $B)$ breast cancer patients $(n=60)$. Dashed lines indicate the mean +1 SD used to indicate the cut-off point. 


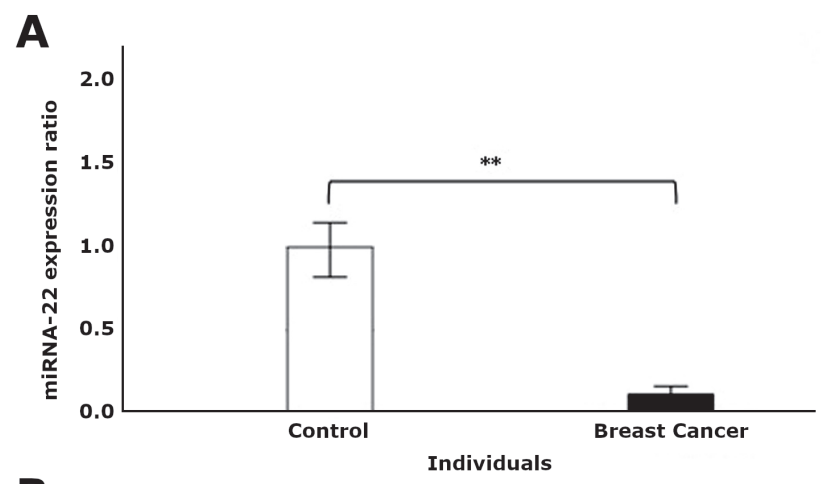

B

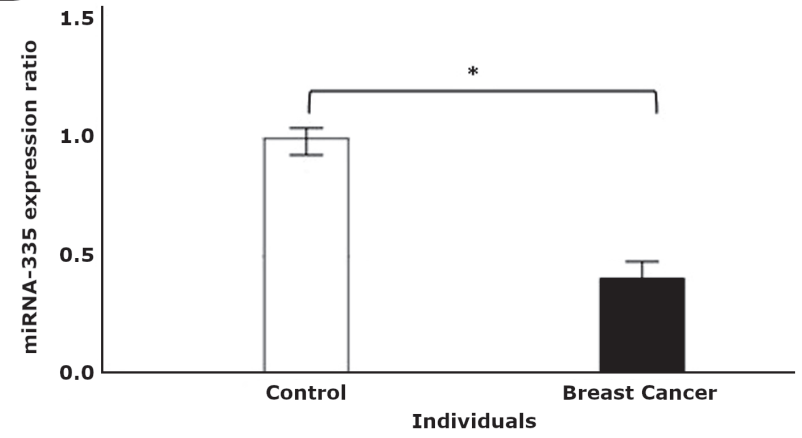

Figure 3. Studied miRNAs analysis in BC A) miR-22 is downregulated in breast cancer ( 60 individuals) compared with healthy individuals (30 individuals). B) miR-335 expression level is reduced in breast cancer (60 individuals) versus healthy control (30 individuals). ${ }^{\star}$ Statistically significant $\mathbf{p}<0.05,{ }^{* *}$ statistically significant $\mathbf{p}<\mathbf{0 . 0 1}$. Error bars indicate standard deviation (SD) from mean values. The data were analyzed using unpaired t-test.

analysis, 5 cases with the least number of chromatid breaks in radiosensitive samples were considered as non-radiosensitive; therefore, the molecular study was finally performed for 2 equal groups of patients with and without cellular radiosensitivity (each with 30 samples).

The association of miR-22 and miR-335 with BC and cellular radiosensitivity. miR-22 was significantly downregulated in PBMCs extracted from 60 BC patients compared with samples taken from 30 healthy individuals (expression ratio $=0.12$, unpaired t-test, $\mathrm{p}=0.006$ ). $\mathrm{miR}-335$ showed also a significantly reduced expression in $60 \mathrm{BC}$ samples in comparison with 30 samples from healthy individuals (expression ratio $=0.41$, unpaired t-test, $\mathrm{p}=0.026$; Figures $3 \mathrm{~A}, 3 \mathrm{~B}$ ).

miR-22 expression was directly associated with cellular radiosensitivity. The expression of this miRNA was reduced in leukocytes of samples with cellular radiosensitivity (expression ratio $=0.17$ ) and without cellular radiosensitivity (expression ratio $=0.06$ ) in comparison with the leukocytes of the control group. It was also found that its expression was significantly higher in the group with cellular radiosensitivity rather than those without cellular radiosensitivity (expres-
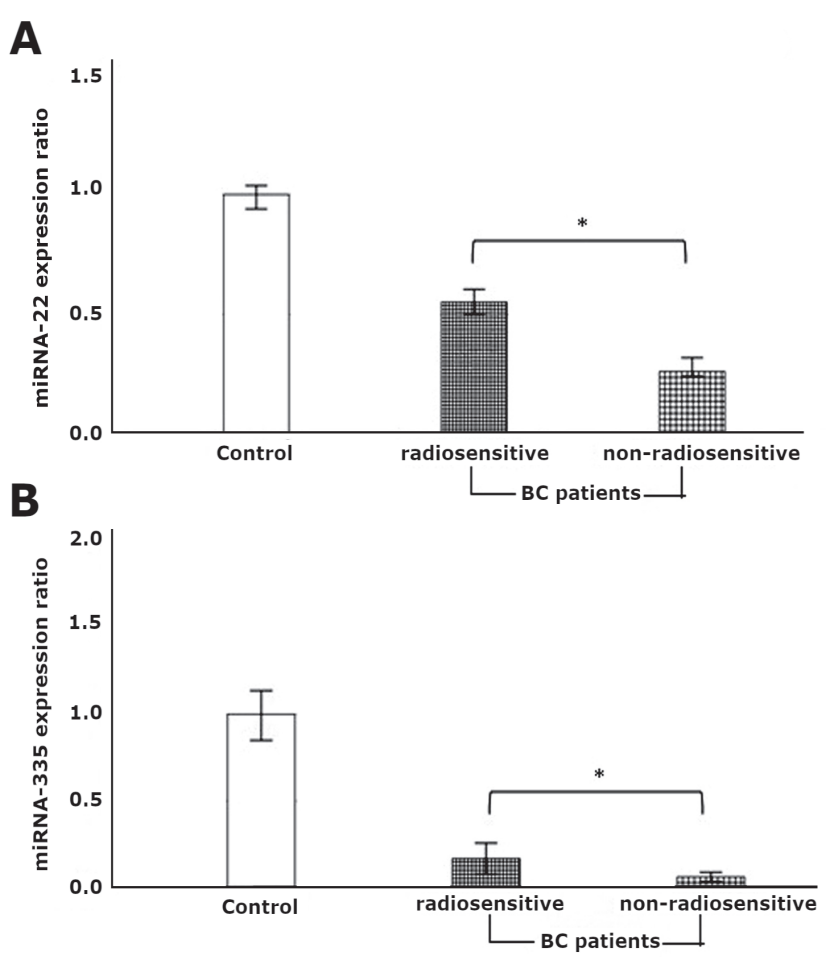

Figure 4. miR-22 and miR-335 expression levels and BC cellular radiation response. Three different groups are indicated in this figure and each of them includes 30 individuals. A) The BC group without cellular radiosensitivity showed the lowest expression level of miR-22. B) The BC group with cellular radiosensitivity showed higher expression of mentioned miRNA compared to the $\mathrm{BC}$ group without cellular radiosensitivity. ${ }^{\star}$ Statistically significant $\mathbf{p}<\mathbf{0 . 0 5}$. Error bars indicate standard deviation (SD) from mean values. One-way ANOVA with post hoc Tukey test was used for data analysis.

sion ratio $=2.83$ ). After performing the Tukey Post Hoc One-way ANOVA test it was found that these observations are statistically significant with respective $\mathrm{p}=0.026, \mathrm{p}=0.001$, $\mathrm{p}=0.036$; Figure 4A).

miR-335 expression analysis among our three studied groups demonstrated that it was also directly related to cellular radiosensitivity in BC patients. A comparison of samples with cellular radiosensitivity and without cellular radiosensitivity with control showed a reduced expression ratio of 0.55 and 0.26 , respectively. A higher expression level was observed in samples with cellular radiosensitivity compared to samples without cellular radiosensitivity (expression ratio = 2.12). Based on Tukey Post Hoc One-way ANOVA test results were statistically significant with $\mathrm{p}=0.049, \mathrm{p}=0.018$, $\mathrm{p}=0.045$, respectively (Figure $4 \mathrm{~B}$ ).

Predictive values of miR-22 and miR-335 in cellular radiosensitivity in $\mathbf{B C}$ patients. Both of miR-22 and miR-335 were differentially expressed in samples taken from BC and healthy individuals, so ROC curves were applied to evaluate the specificity and sensitivity of these miRNAs in discrimination of BC patients and healthy controls. For 
miR-22 a sensitivity of $70 \%$, a specificity of $86.7 \%$, and AUC values 0.810 (0.701-0.919) with 95\% CI was obtained (Figure 5A). Analysis of ROC in miR-335 showed a sensitivity of $86.7 \%$, a specificity of $66.7 \%$, and AUC values 0.743 (0.614-0.873) (Figure 5B). Interestingly it was found that a combination of these two studied miRNAs resulted in enhanced sensitivity $76.7 \%$ and specificity $80 \%$, and AUC value $0.833(0.732-0.935)$ in $\mathrm{BC}$ differentiation from healthy individuals ( $\mathrm{p}=0.0001$, Figure $5 \mathrm{C})$.

ROC curve was also used to evaluate the prediction power of the mentioned miRNAs in cellular radiosensitivity in $\mathrm{BC}$ patients. Regarding radiation response, the AUC values with $95 \%$ CI was $0.756(0.657-0.854 ; \mathrm{p}=0.0003)$, with a sensitivity of $66.7 \%$ and specificity of $70 \%$ for miR-22 (Figure 5D) and $0.723(0.597-0.849 ; \mathrm{p}=0.001)$ with a sensitivity of $66.7 \%$ and specificity of $66.7 \%$ for miR-335 (Figure 5E). Based on obtained results combining miR-22 and miR-335 had promoted sensitivity and specificity in cellular radiosensitivity, since the AUC value reached 0.820 (0.726-0.914) $(\mathrm{p}=0.001)$, with a sensitivity of $80 \%$ and specificity of $66.7 \%$ (Figure 5F).

Binary logistic regression analysis was applied to predict the capability of miR-22 and miR-335 in predicting breast cancer and its cellular radiosensitivity. Obtained results for mir-22 was with Odds Ratio $=4.376(\mathrm{p}=0.002)$ compared to Odds Ratio $=3.263(\mathrm{p}=0.018)$ for miR-335 in BC predication. Odds Ratio index shows the effects of an independent factor on the dependent factor. Therefore, independent factors (for example miR-22) with bigger OR have a more powerful potency in the prediction of dependent factor (such as BC detection). The evaluated results demonstrated that miR-22 was more successful in predicting BC as it could predict BC 1.341 times stronger than miR-335.

Regarding radiation response analysis, evaluation of binary logistic regression indicated only miR-22 with $\mathrm{p}=0.011$ and Odds Ratio $=3.074$ was capable enough to predict cellular radiosensitivity in BC patients, while miR-335 was $\operatorname{not}(\mathrm{p}=0.178)$.

Chromatid breaks frequency in BC molecular subtypes. After determining molecular subtypes of some patients based on pathology reports it was found that $74.46 \%$ of BC cases were HR-positive (luminal A + B), 19.14\% Triple Negative (TN), and $6.38 \%$ HER2+. Based on the results from innate chromosomal aberration analysis (without irradiation) it was found that TN and HER2+ BC subtypes encompass a higher mean of Chromatid Breaks Frequency
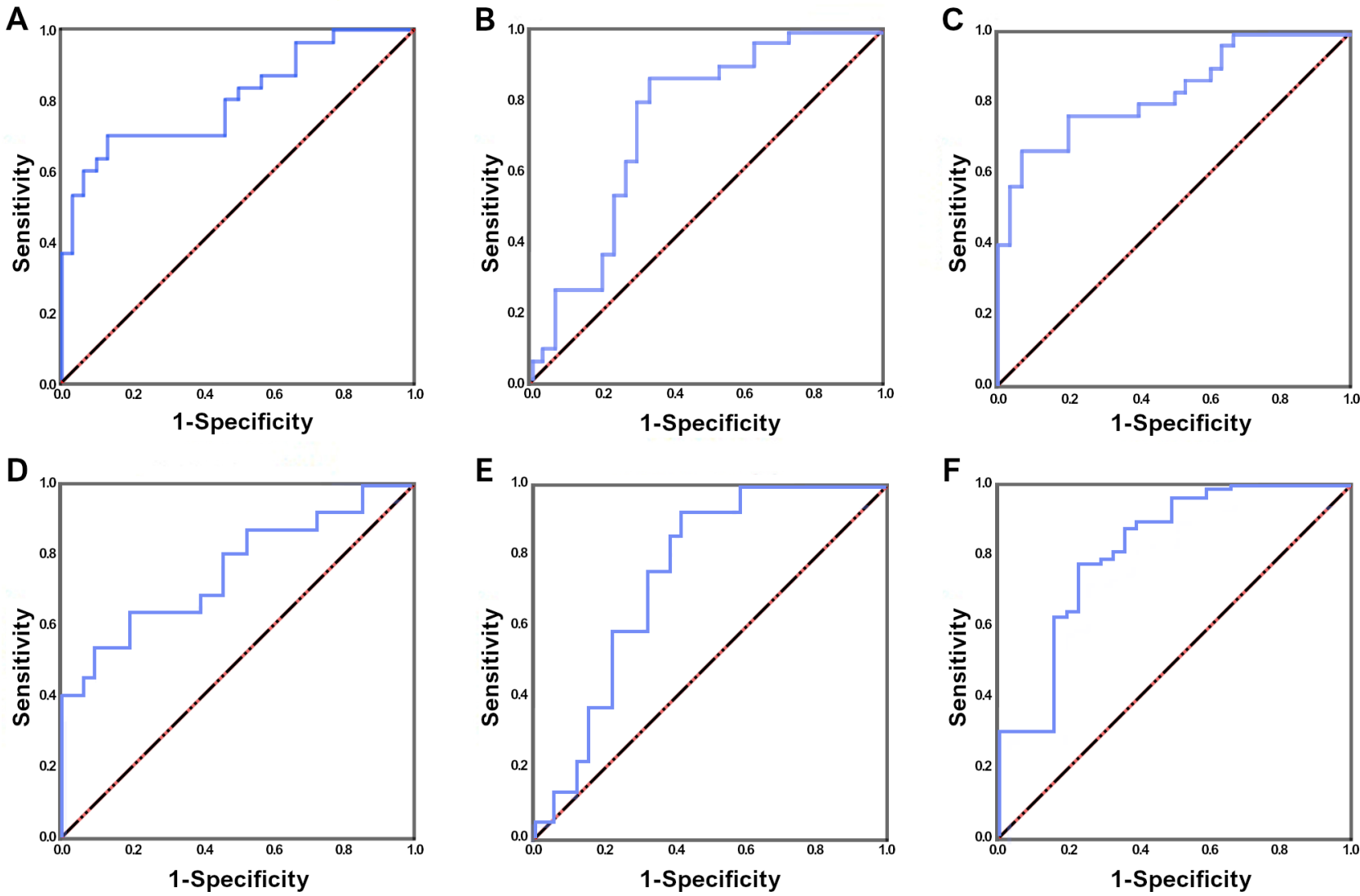

Figure 5. ROC curve analysis A) and B) represent ROC curves of the miR-22 and miR-335 in breast cancer (n=60) compared to healthy controls $(n=30)$ and $C)$ combined ROC curve of both miRNAs. D) and E) represent ROC curves of the miR-22 and miR-335 in patients with cellular radiosensitivity $(n=30)$ compared to patients without cellular radiosensitivity $(n=30)$, and $F)$ combined ROC curve of both miRNAs. 
(CBF) compare to luminal A and B subtypes $(3.67 \pm 1.94$ and $5.33 \pm 1.73$ vs. $3.07 \pm 2.34$ and $3.33 \pm 1.40$, respectively). Luminal A showed the least CBF among all studied subtypes. To assess Radiation Response Score (RRS) among $\mathrm{BC}$ subtypes, induced $\mathrm{CBF}$ was divided by spontaneous $\mathrm{CBF}$ values. A comparison of radiation-induced chromatid breaks with spontaneous chromatid breaks revealed that luminal-B (3.55 \pm 2.74$)$ and HER2+ $(2.43 \pm 1.53)$ had the highest and the lowest RRS, respectively.

Association of miRNA expression ratios with BC molecular subtypes. To evaluate the association of miR-22 and miR-335 expression levels with BC molecular subtypes, a non-parametric Kruskal-Wallis ( $\mathrm{k}$ samples) test was conducted. miRNA expression levels were compared with the control group in each BC molecular sub-types and indicated as "Expression Ratio vs. Control" in Table 2. The expression levels of studied miRNAs were also compared in Triple Positive (TP) versus TN, luminal versus HER2 Positive and luminal A versus luminal $\mathrm{B}$ and obtained results were indicated as "inter-group ratios" in Table 2. These values indicate the magnitude of expression changes in each group in comparison with the others. For instance, $\mathrm{TP}$ has a higher expression of miR-22 rather than the TN group (1.071 times). In summary for miR-22, a significant reduction of expression levels differences was observed in all of the $\mathrm{BC}$ molecular subtypes, compared to control (Kruskal-Wallis test; $\mathrm{p}=0.001$ ). Similar results were obtained for miR-335 except for HER2+ subtypes. miR-335 expression in Triple Positive was significantly higher than the TN subtype (Kruskal-Wallis, $\mathrm{p}=0.013$ ). More details are presented in Table 2.

\section{Discussion}

A probable prospect of radiosensitivity investigations is creating a risk model including genetic assays with the power of anticipating the patient's radiation response and the chance of developing adverse reactions from irradiation [4]. This risk model can eventually be combined with current predictors of radiosensitivity including the dose of radiation, the existence of other diseases or medical conditions in addition to primary cancer, and volume of the target exposed to irradiation [36].

Our results from G2 assays suggested that SY is more frequent in patients than controls $(\mathrm{p}<0.05)$. It was also found that IY was higher in $\mathrm{BC}$ rather than the control group (Figure 2). This result is in line with some previous findings $[9,10,18,37]$.

Furthermore, RIY Scores in BC patients were significantly higher than healthy individuals. On the other hand, lymphocytes of patients were more sensitive to ionizing radiation compared to those of healthy individuals. Inter-individual variation in chromosomal radiosensitivity among $\mathrm{BC}$ patients and healthy individuals were also observed. These results are in agreement with several previous studies on $\mathrm{BC}$ $[9,10,18,19,38-40]$.

Deregulation of miRNAs has been detected in several types of cancer including BC [41]. Studies revealed that miR-22 expression levels are reduced in several types of cancer such as BC [42]. miR-22 was significantly downregulated (0.11 times) in BC-derived PBMCs as shown in Figure 3. It was in agreement with the study done by Xiong et al. in 2010 [42]. Based on our results and according to the fact that miR-22 expression is reduced in several types of cancers (50-53), it can be concluded that miR-22 might probably act as a tumor suppressor miRNA in breast cancer and might be a putative biomarker in the early detection of BC.

Our data revealed that miR-335 expression was significantly downregulated (0.41 times) in PBMCs from BC patients which is in accordance with related studies in $\mathrm{BC}$ $[43,44]$. So, it can be concluded that miR-335 may act as a tumor suppressor miRNA in BC and might be served as a suitable potential biomarker of $\mathrm{BC}$ detection in the early stages.

Table 2. miRNAs expression ratios based on BC molecular subtypes.

\begin{tabular}{|c|c|c|c|c|c|c|}
\hline & BC Molecular Subtypes & Sample Size & $\begin{array}{c}\text { Expression Ratios vs. } \\
\text { Control }\end{array}$ & p-value ${ }^{a}$ & $\begin{array}{c}\text { Inter-group } \\
\text { Ratios }^{b}\end{array}$ & p-value \\
\hline \multirow[t]{6}{*}{ miRNA-22 } & 1. Triple Positive & $7(13 \%)$ & 0.08 & $0.001^{* *}$ & 1.07 & 0.958 \\
\hline & 2. Triple Negative & $9(17 \%)$ & 0.07 & 0.086 & & \\
\hline & 1. Luminal & $35(64 \%)$ & 0.26 & $>0.001^{\star *}$ & 0.30 & 0.317 \\
\hline & 2. HER2 Positive & $3(6 \%)$ & 0.31 & 0.06 & & \\
\hline & 1. Luminal A & $14(26 \%)$ & 0.18 & $0.001^{* *}$ & 0.66 & 0.278 \\
\hline & 2. Luminal B & $21(39 \%)$ & 0.12 & $0.040^{*}$ & & \\
\hline \multirow[t]{6}{*}{ miRNA-335 } & 1. Triple Positive & $7(13 \%)$ & 0.58 & $0.020^{*}$ & 4.8 & $0.013^{*}$ \\
\hline & 2. Triple Negative & $9(17 \%)$ & 0.18 & $>0.001^{\star *}$ & & \\
\hline & 1. Luminal & $35(64 \%)$ & 0.39 & $>0.001^{\star *}$ & 0.49 & 0.223 \\
\hline & 2. HER2 Positive & $3(6 \%)$ & 0.80 & 0.091 & & \\
\hline & 1. Luminal A & $14(26 \%)$ & 0.43 & $0.034^{*}$ & 1.15 & 0.501 \\
\hline & 2. Luminal B & $21(39 \%)$ & 0.37 & $0.004^{* *}$ & & \\
\hline
\end{tabular}

${ }^{a}$ Kruskal-Wallis Non-parametric 2 independent samples test was performed, ${ }^{*}$ statistically significant $\mathrm{p}<0.05$; ${ }^{\text {b }}$ to obtain the expression ratio of miRNAs in each group, the average of miRNA expression in group 1 was calculated and then divided by group 2 , such as the expression level in TP (group 1 ) was divided by Triple Negative (group 2) 
Exposure to ionizing radiation significantly alters miRNA expression patterns in normal cells as well as in cancer cells $[45,46]$. Studies showed that the overexpression of miR-22 could significantly increase the number of chromatids breaks as compared with control cells [47]. As shown in Figure 4, the expressions level of miR-22 in the radiosensitive group was significantly higher than the non-radiosensitive group (2.83 times) which may be due to restraining DNA damage repair by the miR-22 overexpression [48]. These findings are in line with the results of other studies indicating an association of miR-22 with radiosensitivity [30, 32]. Moreover, the expression level of miR-335 in the radiosensitive group was significantly higher than the non-radiosensitive group (2.11 times) in line with other reports $[32,43]$. Tavazoie et al. found that the lower expression of miR-335 might potentially cause more resistance to irradiation in breast cancer cells [43]. Based on the obtained results, the expression changes of these two miRNAs may be considered as potential biomarkers in cellular radiosensitivity in BC patients which might help physicians in determining radiosensitive patients before RT more efficiently in future that need to be confirmed in clinical studies.

Plotting ROC curves for miR-22 and miR-335 also indicated an acceptable specificity and sensitivity for breast cancer differentiation from healthy individuals (Figure 5). Combined miR-22 and miR-335 ROC curves identified slightly improved AUC value, suggesting that evaluation of these two miRNAs simultaneously, encompass a more powerful differentiation of $\mathrm{BC}$ from control groups. Moreover, both miR-22 and miR-335 were able in the separation of radiosensitive lymphocytes of $\mathrm{BC}$ patients from non-radiosensitive ones with suitable specificity and sensitivity. Combined ROC analysis revealed an enhanced AUC value, which is an indicator of their additive effect in cellular radiosensitivity detection. Binary logistic regression also confirmed the ability of miR-22 and miR-335 in determining of BC patients from healthy volunteers. Both of the miRNAs were able to predict breast cancer, however, miR-22 was a stronger predictor. In cellular radiosensitivity analysis, only miR-22 was strong enough to predict the radiosensitivity of $\mathrm{BC}$ derived lymphocytes successfully.

Comparison of spontaneous CBF among different breast cancer subtypes revealed that HER2+ and TNBC subtypes had a higher CBF rather than luminal subtypes. Since both HER2+ and TNBC patients have a poor prognosis and the luminal ones have a better prognosis, our obtained results seem logical. We found that the luminal-A group had the lowest spontaneous CBF among all studied groups. Provided that the luminal-A group generally has a better prognosis than other subtypes and also, they have a better response to RT, the obtained results are in agreement with our expectations. Besides, the analysis of radiation response revealed that luminal-B had the highest (3.55 times) and HER2+ had the lowest (2.43 times) radiation response among all subtypes. Our data revealed that the number of non-radiosensitive patients was higher than the radiosensitive ones in the TN group. Although in luminal-A and -B subtypes, most of the members were radiosensitive. These results are in accordance with some studies [49].

As shown in Table 2, analysis of miRNA expression ratios in different $\mathrm{BC}$ molecular subtypes compared to healthy individuals revealed that miR-22 had a higher expression in $\mathrm{BC}$ molecular subtypes with a better prognosis except for its results in HER2+ versus luminal groups. Interestingly, Farazi et al. found that miR-22 had a higher expression in HER2+ and luminal-B subtypes which is in accordance with our results. It refers to the fact in some cases miR-22 acts as an oncomiR [50]. Analysis of miR-335 expression ratios in different $\mathrm{BC}$ molecular subtypes compared to healthy individuals revealed that it had a higher expression in $\mathrm{BC}$ molecular subtypes with a better prognosis. It shows that in some cases miR-335 can act as an oncomiR which is in agreement with some studies [51].

Based on clinicopathological information of the participants in each studied group, the number of individuals in each subtype was inevitably unequal. To achieve more precise results in the association study of these miRNAs with BC subtypes, investigation of miRNAs expression levels in association with radiosensitivity in a larger sample size might seem necessary. To obtain the clinical utility of these findings further clinical studies are recommended.

In conclusion, miR-22 and miR-335 downregulation indicate that they might act as tumor suppressor miRNAs in breast cancer. miR-22 and miR-335 may be considered as possible biomarkers in breast cancer detection. As both miRNAs were associated with cellular radiosensitivity they might be the promising potential biomarkers in determining cellular radiosensitivity in BC patients in vitro, which needs to be confirmed in other clinical studies for future applications in the detection of $\mathrm{BC}$ radiosensitivity.

Acknowledgments: This study was supported by a grant (grant number: IG-39711) from the Research Department of the Tarbiat Modares University, Tehran, Iran. The authors sincerely appreciate the head and staff of the oncology departments of Cancer Institute Imam Khomeini hospital for their valuable cooperation. The authors also thank all patients and healthy volunteers for their involvement in our study. We also appreciate Mr. H. Nosrati for the irradiation of the samples.

\section{References}

[1] JEMAL A, BRAY F, CENTER MM, FERLAY J, WARD E et al. Global cancer statistics. CA Cancer J Clin 2011; 61: 69-90. https://doi.org/10.3322/caac.20107

[2] DELANEY G, JACOB S, FEATHERSTONE C, BARTON $M$. The role of radiotherapy in cancer treatment: estimating optimal utilization from a review of evidence-based clinical guidelines. Cancer 2005; 104: 1129-1137. https://doi. org/10.1002/cncr.21324 
[3] EARLY BREAST CANCER TRIALISTS' COLLABORATIVE GROUP (EBCTCG), DARBY S, MCGALE P, CORREA C, TAYLOR C et al. Effect of radiotherapy after breastconserving surgery on 10-year recurrence and 15-year breast cancer death: meta-analysis of individual patient data for 10 801 women in 17 randomised trials. Lancet 2011; 378: 17071716. https://doi.org/10.1016/S0140-6736(11)61629-2

[4] RATTAY T, TALBOT C. Finding the genetic determinants of adverse reactions to radiotherapy. Clin Oncol (R Coll Radiol) 2014; 26: 301-308. https://doi.org/10.1016/j. clon.2014.02.001

[5] WEST CM, BARNETT GC. Genetics and genomics of radiotherapy toxicity: towards prediction. Genome Med 2011; 3: 52. https://doi.org/10.1186/gm268

[6] KUO SH, HUANG CS. Association between radiosensitivity and molecular subtypes in patients with early-stage breast cancer and lymph node-negative status. Translational Cancer Research 2017; S1462-S1466. https://doi.org/10.21037/ tcr.2017.11.09

[7] MEATTINI I, FRANCOLINI G, LIVI L. Radiosensitivity in the breast cancer management scenario: another step forward? J Thorac Dis 2016; 8: E1361-E1363. https://doi. org/10.21037/jtd.2016.10.24

[8] DE RUYCK K, DE GELDER V, VAN EIJKEREN M, BOTERBERG T, DE NEVE W et al. Chromosomal radiosensitivity in head and neck cancer patients: evidence for genetic predisposition? Br J Cancer 2008; 98: 1723-1738. https://doi.org/10.1038/sj.bjc.6604345

[9] SCOTT D, BARBER JBP, SPREADBOROUGH AR, BURRIL W, ROBERTS SA. Increased chromosomal radiosensitivity in breast cancer patients: a comparison of the two assays. Int J Radiat Biol 1999; 75: 1-10. https://doi. org/10.1080/095530099140744

[10] BAEYENS A, THIERENS H, CLAES K, POPPE B, MESSIAEN L et al. Chromosomal radiosensitivity in breast cancer patients with a known or putative genetic predisposition. Br J Cancer 2002; 87: 1379-1385. https://doi.org/10.1038/ sj.bjc. 6600628

[11] TERZOUDI GI, HATZI VI, BARSZCZEWSKA K, MANOLA KN, STAVROPOULOU C et al. G2-checkpoint abrogation in irradiated lymphocytes: A new cytogenetic approach to assess individual radiosensitivity and predisposition to cancer. Int J Oncol 2009; 35: 1223-1230. https://doi. org/10.3892/ijo_00000439

[12] PARSHAD R, SANFORD KK, JONES GM. Chromatid damage after G2 phase X-irradiation of cells from cancerprone individuals implicates deficiency in DNA repair. Proc Natl Acad Sci U S A 1983; 80: 5612-5626. https://doi. org/10.1073/pnas.80.18.5612

[13] MOZDARANI H, BRYANT PE. Kinetics of chromatid aberrations in G2 ataxia-telangiectasia cells exposed to X-rays and ara A. Int J Radiat Biol 1989; 55: 71-84. https://doi. org/10.1080/09553008914550081

[14] MAHMOODI M, ABOLHASSANI H, MOZDARANI $\mathrm{H}$, REZAEI N, AZIZI G et al. In vitro chromosomal radiosensitivity in patients with common variable immunodeficiency. Cent Eur J Immunol 2018; 43: 155-161. https://doi. org/10.5114/ceji.2018.77385
[15] Mozdarani H, Kiaee F, Fekrvand S, Azizi G, Yazdani R et al. G2-lymphocyte chromosomal radiosensitivity in patients with LPS responsive beige-like anchor protein (LRBA) deficiency. Int J Radiat Biol 2019; 95: 680-690. https://doi.org/10 $.1080 / 09553002.2019 .1577570$

[16] Amirifar P, Mozdarani H, Yazdani R, Kiaei F, Moeini Shad $\mathrm{T}$ et al. Effect of Class Switch Recombination Defect on the Phenotype of Ataxia-Telangiectasia Patients. Immunol Invest 2020; 1-15. https://doi.org/10.1080/08820139.2020.172 3104

[17] SCOTT D. Chromosomal radiosensitivity, cancer predisposition and response to radiotherapy. Strahlenther Onkol 2000; 176: 229-234. https://doi.org/10.1007/s000660050005

[18] MOZDARANI H, ZIAEE MASHHADI AH, ALIMOHAMMADI Z. G2 chromosomal radio sensitivity and background frequency of sister chromatid exchanges of peripheral blood lymphocytes of breast cancer patients. Int J Radiat Res 2011; 9: 167-174.

[19] MOZDARANI H, SALIMI M, BAKHTARI N. Inherent radiosensitivity and its impact on breast cancer chemo-radiotherapy. Int. J. Radiat. Res., 2017; 15(4): 325-341.

[20] VIGNARD J, MIREY G, SALLES B. Ionizing-radiation induced DNA double-strand breaks: a direct and indirect lighting up. Radiother Oncol 2013; 108: 362-369. https://doi. org/10.1016/j.radonc.2013.06.013

[21] LÖBRICH M, JEGGO P. A Process of Resection-Dependent Nonhomologous End Joining Involving the Goddess Artemis. Trends Biochem Sci 2017; 42: 690-701. https://doi. org/10.1016/j.tibs.2017.06.011

[22] BRYANT PE. Mechanisms of radiation-induced chromatid breaks. Mutat Res 1998; 404: 107-111. https://doi. org/10.1016/s0027-5107(98)00101-8

[23] BRYANT PE, MOZDARANI H. Mechanisms underlying the conversion of DNA double-strand breaks into chromatid breaks. Mutat Res 2010; 701: 23-26. https://doi. org/10.1016/j.mrgentox.2010.03.016

[24] LIU X, LI F, HUANG Q, ZHANG Z, ZHOU L et al. Selfinflicted DNA double-strand breaks sustain tumorigenicity and stemness of cancer cells. Cell Res 2017; 27: 764-783. https://doi.org/10.1038/cr.2017.41

[25] JACKSON SP, BARTEK J. The DNA-damage response in human biology and disease. Nature 2009; 461: 1071-1078. https://doi.org/10.1038/nature08467

[26] VERGHESE ET, HANBY AM, SPEIRS V, HUGHES TA. Small is beautiful: microRNAs and breast cancer-where are we now? J Pathol 2008; 215: 214-221. https://doi. org/10.1002/path.2359

[27] LEE JH, PARK SJ, KIM SW, HARIHARASUDHAN G, JUNG SM et al. c-Fos-dependent miR-22 targets MDC1 and regulates DNA repair in terminally differentiated cells. Oncotarget 2017; 8: 48204-48221. https://doi.org/10.18632/ oncotarget.18389

[28] BARTKOVA J, HOŘEJSí Z, SEHESTED M, NESLAND J, RAJPERT-DE MEYTS E et al. DNA damage response mediators MDC1 and 53BP1: constitutive activation and aberrant loss in breast and lung cancer, but not in testicular germ cell tumours. Oncogene 2007; 26: 7414-7422. https://doi. org/10.1038/sj.onc. 1210553 
[29] YANG C, NING S, LI Z, QIN X, XU W. miR-22 is downregulated in esophageal squamous cell carcinoma and inhibits cell migration and invasion. Cancer Cell Int 2014; 14: 138. https://doi.org/10.1186/s12935-014-0138-0

[30] ZHANG X, LI Y, WANG D, WEI X. miR-22 suppresses tumorigenesis and improves radiosensitivity of breast cancer cells by targeting Sirt1. Biol Res 2017; 50: 27. https://doi. org/10.1186/s40659-017-0133-8

[31] CHEN B, TANG H, LIU X, LIU P, YANG L et al. miR-22 as a prognostic factor targets glucose transporter protein type 1 in breast cancer. Cancer Lett 2015; 356: 410-417. https://doi. org/10.1016/j.canlet.2014.09.028

[32] VARES G, CUI X, WANG B, NAKAJIMA T, NENOI M. Generation of breast cancer stem cells by steroid hormones in irradiated human mammary cell lines. PLoS One 2013; 8: e77124. https://doi.org/10.1371/journal.pone.0077124

[33] MARTIN NT, NAKAMURA K, DAVIES R, NAHAS SA, BROWN C et al. ATM-dependent miR-335 targets CtIP and modulates the DNA damage response. PLoS Genet 2013; 9: e1003505. https://doi.org/10.1371/journal.pgen.1003505

[34] YUN MH, HIOM K. CtIP-BRCA1 modulates the choice of DNA double-strand-break repair pathway throughout the cell cycle. Nature 2009; 459: 460-463. https://doi. org/10.1038/nature07955

[35] HEYN H, ENGELMANN M, SCHREEK S, AHRENS P, LEHMANN U et al. MicroRNA miR-335 is crucial for the BRCA1 regulatory cascade in breast cancer development. Int J Cancer 2011; 129: 2797-2806. https://doi.org/10.1002/ ijc. 25962

[36] BARNETT G, WILKINSON J, MOODY A, WILSON C, TWYMAN N et al. The Cambridge Breast Intensity-modulated Radiotherapy Trial: patient-and treatment-related factors that influence late toxicity. Clin Oncol (R Coll Radiol) 2011; 23: 662-673. https://doi.org/10.1016/j.clon.2011.04.011

[37] ERNESTOS B, NIKOLAOS P, KOULIS G, ELENI R, KONSTANTINOS B et al. Increased chromosomal radiosensitivity in women carrying BRCA1/BRCA2 mutations assessed with the G2 assay. Int J Radiat Oncol Biol Phys 2010; 76: 1199-1205. https://doi.org/10.1016/j.ijrobp.2009.10.020

[38] Terzoudi ITJ, Hain J, Vrouvas J, Margaritis K, Donta-Bakoyianni C et al. Increased G2 chromosomal radiosensitivity in cancer patients: the role of cdk1/cyclin-B activity level in the mechanisms involved. Int J Radiat Biol 2000; 76: 607-615. https://doi.org/10.1080/095530000138268

[39] Riches A, Bryant P, Steel C, Gleig A, Robertson A et al. Chromosomal radiosensitivity in G 2-phase lymphocytes identifies breast cancer patients with distinctive tumour characteristics. Br J Cancer 2001; 85: 1157-1161. https://doi. org/10.1054/bjoc.2001.2086

[40] SCOTT D, SPREADBOROUGH A, LEVINE E, ROBERTS SA. Genetic predisposition in breast cancer. Lancet 1994; 344: 1444. https://doi.org/10.1016/s0140-6736(94)90615-7
[41] WANG W, LUO YP. MicroRNAs in breast cancer: oncogene and tumor suppressors with clinical potential. J Zhejiang Univ Sci B 2015; 16: 18-31. https://doi.org/10.1631/jzus. B1400184

[42] XIONG J, YU D, WEI N, FU H, CAI T et al. An estrogen receptor alpha suppressor, microRNA-22, is downregulated in estrogen receptor alpha-positive human breast cancer cell lines and clinical samples. FEBS J 2010; 277: 1684-1694. https://doi.org/10.1111/j.1742-4658.2010.07594.x

[43] TAVAZOIE SF, ALARCÓN C, OSKARSSON T, PADUA D, WANG Q et al. Endogenous human microRNAs that suppress breast cancer metastasis. Nature 2008; 451: 147-152. https://doi.org/10.1038/nature06487

[44] WANG F, ZHENG Z, GUO J, DING X. Correlation and quantitation of microRNA aberrant expression in tissues and sera from patients with breast tumor. Gynecol Oncol 2010; 119: 586-593. https://doi.org/10.1016/j.ygyno.2010.07.021

[45] CHAUDHRY MA, KREGER B, OMARUDDIN RA. Transcriptional modulation of micro-RNA in human cells differing in radiation sensitivity. Int J Radiat Biol 2010; 86: 569583. https://doi.org/10.3109/09553001003734568

[46] WEIDHAAS JB, BABAR I, NALLUR SM, TRANG P, ROUSH S et al. MicroRNAs as potential agents to alter resistance to cytotoxic anticancer therapy. Cancer Res 2007; 67: 11111-11116. https://doi.org/10.1158/0008-5472.CAN-072858

[47] LEE JH, PARK SJ, JEONG SY, KIM MJ, JUN S et al. MicroRNA-22 Suppresses DNA Repair and Promotes Genomic Instability through Targeting of MDC1. Cancer Res 2015; 75: 1298-1310. https://doi.org/10.1158/0008-5472.CAN-142783

[48] ZHANG X, LI Y, WANG D, WEI X. miR-22 suppresses tumorigenesis and improves radiosensitivity of breast cancer cells by targeting Sirt1. Biol Res 2017; 50: 27. https://doi. org/10.1186/s40659-017-0133-8

[49] BORGMANN K, RÖPER B, EL-AWADY RA, BRACKROCK S, BIGALKE $\mathrm{M}$ et al. Indicators of late normal tissue response after radiotherapy for head and neck cancer: fibroblasts, lymphocytes, genetics, DNA repair, and chromosome aberrations. Radiother Oncol 2002; 64: 141-152. https://doi. org/10.1016/s0167-8140(02)00167-6

[50] FARAZI TA, TEN HOEVE JJ, BROWN M, MIHAILOVIC A, HORLINGS HM et al. Identification of distinct miRNA target regulation between breast cancer molecular subtypes using AGO2-PAR-CLIP and patient datasets. Genome Biol 2014; 15: R9. https://doi.org/10.1186/gb-2014-15-1-r9

[51] MARTIN EC, CONGER AK, YAN TJ, HOANG VT, MILLER DF et al. MicroRNA-335-5p and -3p synergize to inhibit estrogen receptor alpha expression and promote tamoxifen resistance. FEBS Lett 2017; 591: 382-392. https://doi. org/10.1002/1873-3468.12538 